\title{
FATORES QUE DIFICULTAM A ADESÃO DE MULHERES AO EXAME PAPANICOLAU
}

\begin{abstract}
Maria Ivanilde de Andrade*1, Pamela Nery do Lago*2, Maria Fernanda Silveira Scarcella3, Karine Alkmim Durães ${ }^{4}$, Lilian Maria Santos Silva ${ }^{5}$, Aline Francielly Rezende Fróes ${ }^{5}$, Milenny Andreotti e Silva ${ }^{5}$, Adriano Ferreira de Oliveira ${ }^{5}$, Veridiana de Oliveira Ursi Scotton ${ }^{5}$, Edmilson Escalante Barboza $^{5}$, Irismar Emília de Moura Marques ${ }^{5}$, Jéssica de Oliveira dos Anjos ${ }^{6}$, Edma Nogueira da Silva7, Diélig Teixeira ${ }^{8}$, Glauber Marcelo Dantas Seixas ${ }^{9}$, Emanoel Rodrigo Melo dos Santos $^{10}$, Manuela Amaral Almeida Costa11, Ira Caroline de Carvalho Sipoli12, Milena Vaz Sampaio Santos ${ }^{12}$, Fernanda Carneiro Melo ${ }^{13}$, Ítalo Freire Cantalice ${ }^{14}$, Pedro Henrique Santos Oliveira $^{14}$ and Fernando Henrique da Silva Costa ${ }^{14}$
\end{abstract}

1Enfermeira Mestre da Estratégia Saúde da Família do Município de Lagoa Santa-MG e da Clínica Médica do Hospital das Clínicas da Universidade Federal de Minas Gerais pela Empresa Brasileira de Serviços Hospitalares (CLM/HCUFMG/EBSERH); ${ }^{2}$ Enfermeira Mestranda em Gestão de Cuidados da Saúde e Coordenadora de Enfermagem da CLM/HCUFMG/EBSERH; ${ }^{3}$ Enfermeira Doutoranda em Ciências da Saúde pela Universidade Estadual de Montes Claros, Docente do Departamento de Enfermagem da Faculdade Pitágoras de Contagem e Enfermeira da CLM/HC-UFMG/EBSERH;4Enfermeira da CLM/HC-UFMG/EBSERH; ${ }^{5}$ Enfermeiro(a) do Hospital Universitário Maria Aparecida Pedrossian da Universidade Federal do Mato Grosso do Sul (HUMAP-UFMS/EBSERH); ${ }^{6}$ Enfermeira Residente em Enfermagem Obstétrica pela UFMS; ${ }^{7}$ Enfermeira do Hospital Universitário da Universidade Federal de Juiz de Fora (HU-UFJF/EBSERH); ${ }^{8}$ Enfermeiro do Hospital Adventista de Belém (HAB); ${ }^{9}$ Enfermeiro da Fundação Santa Casa de Misericórdia do Pará (FSCMPA); ${ }^{10}$ Farmacêutico-bioquímico do Complexo Hospitalar Universitário da Universidade Federal do Pará (CHU-UFPA/EBSERH); ${ }^{11}$ Enfermeira do Hospital Universitário Professor Alberto Antunes da Universidade Federal de Alagoas (HUPAA/EBSERH); ${ }^{12}$ Enfermeira do Hospital Universitário de Brasília (HUB-UNB/EBSERH); ${ }^{13}$ Enfermeira Mestre do Hospital Universitário da Universidade Federal de Sergipe (HU-UFS/EBSERH); ${ }^{14}$ Acadêmico de Medicina pelo Centro Universitário UNIFACISA.

\section{ARTICLE INFO}

\section{Article History:}

Received $14^{\text {th }}$ March, 2021

Received in revised form

$06^{\text {th }}$ April, 2021

Accepted 03 ${ }^{\text {rd }}$ May, 2021

Published online $30^{\text {th }}$ June, 2021

\section{Key Words:}

Esfregaço vaginal, Saúde da mulher,

Teste de Papanicoloau,

Cuidados de enfermagem.

*Corresponding author:

Maria Ivanilde de Andrade

\begin{abstract}
Objetivo: Identificar os principais fatores que dificultam a adesão de mulheres ao exame Papanicolau. Método: Trata-se de um estudo descritivo, realizado através de uma revisão de literatura, cujo tema abordou os fatores que predispõem a não adesão de mulheres ao exame Papanicolau. Foram utilizados dez artigos científicos, publicados entre 2019 e 2021, com busca na Biblioteca Virtual em Saúde (BVS), sites e plataformas científicas. Resultados: Fatores como medo, vergonha, preconceito, falta de tempo, falta de conhecimento acerca do exame e da vacina, negação, falta de vínculo, insegurança, falta de ética dos profissionais, falta de estrutura são os principais fatores que levam a não adesão ao exame. Conclusão: $O$ câncer do colo de útero tem se tornado um problema de saúde pública, o que nos remete a pensar em estratégias para minimizar este quadro. Esta patologia pode ser diagnosticada precocemente através do exame Papanicolau que é considerado um exame de grande valia e baixo custo, mas para que este exame se torne efetivo entre as mulheres faz se necessário, juntamente com as políticas de saúde, o conhecimento destes fatores bem como rompimento das barreiras que impedem a adesão de mulheres ao exame preventivo.
\end{abstract}

Copyright (C) 2021, Maria Ivanilde de Andrade et al. This is an open access article distributed under the Creative Commons Attribution License, which permits unrestricted use, distribution, and reproduction in any medium, provided the original work is properly cited.

Citation: Maria Ivanilde de Andrade, Pamela Nery do Lago, Maria Fernanda Silveira Scarcella, Karine Alkmim Durães, et al. 2021. "Fatoresque dificultam a adesãodemulheresao examepapanicolau", International Journal of Development Research, 11, (06), 48023-48026.

\section{INTRODUCTION}

Mundialmente o câncer de colo de útero $(\mathrm{CCU})$ é considerado o quarto tipo de câncer mais frequente entre as mulheres. Aproximadamente $80 \%$ dos casos ocorrem em regiões menos desenvolvidas (Garcia et al., 2020).
Estudos apontam que em 2018, ocorreram cerca de 570.000 casos e 311.000 mortes por CCU em todo o mundo. No Brasil, de acordo com o Ministério da Saúde (MS), houve uma previsão para o aparecimento de aproximadamente 16.370 novos casos entre os anos de 2018 e 2019, representando uma incidência de 15,43 casos para cada 100 mil mulheres, com maior ocorrência na região norte do País (Mesquita et al., 2020; Monteiro, Andrade \& Vargas, 2019). 
Devido suas altas taxas de incidência e mortalidade, o CCU é considerado um problema de saúde pública no Brasil, sendo a terceira neoplasia que mais atinge as mulheresa partir dos 30 anos de idade (Mesquita et al., 2020; Garcia et al., 2020; Alencar, Mendes \& Carvalho, 2020). Apesar da alta incidência e mortalidade, o CCU se configura como um câncer com possibilidade de prevenção, e com estratégias de prevenção consolidadas nas políticas de atenção a saúde (Silva, Marques \& Costa, 2020). O CCU se desenvolve a partir de lesões primárias que não foram detectadas ou tratadas corretamente. O principal fator de risco para o desenvolvimento do CCU é o Papilomavírus Humano (HPV), particularmente, os de alto risco oncogênico, sendo os mais frequentes o HPV 16 e o 18 que podem ser detectados em 99,7\% dos casos confirmados (Mesquita et al., 2020; Garcia et al., 2020; Monteiro, Andrade \& Vargas, 2019). Para Oliveira et al. (2020), a descoberta do HPV como causa necessária para o desenvolvimento do CCU tem levado estudiosos e governo ao desenvolvimento de vacinas profiláticas contra o vírus HPV e a ampliação de programas de rastreio e controle do câncer.Entretanto, além do HPV, o câncer de colo do útero possui outros fatores de riscos adicionais, comoo início precoce da prática sexual, multiplicidade e promiscuidade de parceiros sexuais, histórico de doenças sexualmente transmissíveis, número de gestações, fatores imunológicos, tabagismo, nível sócio econômico, uso de contraceptivos orais, entre outros(Melo et al., 2021; Mesquita et al., 2020; Monteiro, Andrade \& Vargas, 2019).

A principal forma de detecção precoce do CCU é através do diagnóstico precoce, realizado através do exame Papanicolau ou exame preventivo de câncer de colo do útero (PCCU). Porém, mesmo sendo um exame simples e de fácil realização,uma parcela considerável das mulheres não aderem à realização e periodicidade do mesmo (Mesquita et al., 2020; Garcia et al., 2020; Alencar, Mendes \& Carvalho, 2020). Diante dessa constatação, torna de extrema importância o papel do profissional de saúde, frente a ações de prevenção da doença, uma vez que esse profissional deve agir como facilitador para promover a realização do exame PCCU e incentivar sua prática, bem como demostrar sua importância, através da identificação e minimização dos fatores que podem servir de barreira para a sua não realização (Mesquitaet al., 2020). Ainda relacionado à detecção precoce do CCU, Marques e Paiva (2019) afirmam que o Sistema Único de Saúde (SUS) tem como principal ferramenta a Atenção Primária à Saúde (APS), por meio das Unidades Básicas de Saúde (UBS), as quais são a porta de entrada para o sistema. Essas mesmas autoras afirmam que a APS possui, entre as suas diversas abordagens, o desenvolvimento de ações para a prevenção do CCU, visto que está mais próximo do cotidiano das mulheres e as acompanham ao longo de suas vidas (Marques \& Paiva, 2019).

Nessa direção, Oliveira et al.(2020), destacam como formas de prevenção primária do CCU ações comportamentais como vacinação contra o HPV, uso de preservativo, redução do número de parceiros sexuais e atraso no início da vida sexual. Como prevenção secundária, esses mesmos autores informam que o exame citopatológico é o mais utilizado, pois o CCU tem progressão lenta e se descoberta a lesão pré-invasiva precocemente, a chance de cura é quase total. Corroborando com Oliveira et al., Marques e Paiva (2019) reforçam que a maior parte das ações de prevenção ao CCU ocorre na APS. No qual:

Tanto ações de rastreamento, que consistem em realizar sistematicamente testes ou exames em pessoas sadias, quanto às ações de diagnóstico precoce, que consistem em captar precocemente alguém que já tenha sintomas ou alterações no exame físico; devem ser realizadas no cotidiano das equipes (Marques \& Paiva, 2019, p. 985).

Quanto à prevenção do CCU, Silva, Marques e Costa (2020) alertam que ações estão sendo criadas, mas os números continuam aumentando, e a mortalidade permanece em platô, o que indica que há barreiras além do aspecto político que devem ser analisadas.

Diante disso, o conhecimento das mulheres sobre os fatores causadores e a forma de detecção precoce do CCU torna-se indispensável e pode contribuir para que as mesmas possam participar das ações e decisões que afetam sua saúde, principalmente estimulando a identificação de sintomas e a procura pelo serviço de prevenção adequado (Oliveiraet al., 2020). Compreendendo a importância das ações de prevenção ao CCU e do acesso à sua realização, bem como a identificação do conhecimento das mulheres frente essa a essa temática, levantou-se o seguinte questionamento: que fatores dificultam a adesão de mulheres à realização do exame preventivo ou Papanicolau?

Frente ao que foi exposto, o objetivo deste estudo foi identificar fatores dificultadores ou que levam a não adesão de mulheres à realização do exame preventivo ou Papanicolau.

\section{MATERIAIS E MÉTODOS}

Trata-se de um estudo de revisão de literatura, de cunho descritivo qualitativo (Marconi\& Lakatos, 2017) extraído da Biblioteca Virtual em Saúde (BVS), sites e plataformas científicas, utilizando-se os seguintes descritores: esfregaço vaginal, saúde da mulher, teste de Papanicoloau, cuidados de enfermagem. Para tanto, as pesquisas em artigos científicos sobre o tema aconteceram no período de abril a maio de 2021. Para construção do mesmo foi analisado e lido na íntegra 25 artigos científicos, sendo eleitos 10 de relevância para o desenvolvimento do tema. Foi considerado como critério de inclusão artigos completos publicados no período de 2019 e 2021, cujos objetivos viessem de encontro ao problema da pesquisa. Foram excluídas as literaturas com mais de 3 anos de publicação e que não contribuísse para a pesquisa.

Para seleção do material foram analisados e selecionados com base nos títulos e posteriormente nos resumos, visando analisar os fatores dificultadores ou que levassem a não adesão das mulheres a realização do exame de Papanicoloau.Por fim, realizada a análise dos dados coletados para o desenvolvimento do mesmo e elaboração das considerações finais acerca do presente estudo, instituindo consonância com os objetivos fundamentados (Marconi\& Lakatos, 2017).

\section{RESULTADOS E DISCUSSÃO}

O quadro 1 mostra os fatores dificultadores da adesão de mulheres ao exame Papanicolau. Embora se tenha conhecimento técnico sobre estratégias de prevenção para fornecer um dos mais altos potenciais de cura, Santos, Batista e Oliveira (2020) alertam que a neoplasia cérvico uterina ainda possui elevada taxa de mortalidade, permanecendo como um problema de saúde pública no Brasil. O CCU tem desenvolvimento lento e silencioso, inicialmente sendo observada a neoplasia intraepitelial cervical. Sabidamente a infecção pelo HPV é uma condição necessária para sua ocorrência (Silva, Marques \& Costa, 2020).

Santos, Batista e Oliveira (2020) afirmam que o pico de incidência do CCU ocorre entre mulheres de 40 aos 49 anos e uma pequena porcentagem em mulheres com menos de 30 anos. Essas mesmas autoras defendem também quea faixa etária para detecção precoce é dos 20 aos 29 anos, período que corresponde ao pico de incidências das lesões precursoras da doença.

A taxa de mortalidade é elevada. O carcinoma in situ tem seu ápice de incidência em mulheres entre 25 e 40 anos, enquanto o carcinoma invasor entre 48 e 55 anos, deve se lembrar do caráter lento desse carcinoma, a evolução natural para câncer invasor são 10 anos, mas a média é 30 anos (Silva, Marques \& Costa, 2020, p. 61).

O HPV é o principal fator etiológicoe o patógeno viral sexual mais comumdo CCU (causado através da infecção por subtipos oncogênicos do HPV). Sua etiologia está associada aos hábitos de vida, aos fatores ambientais, baixas condições socioeconômicas e a 


\section{QUADRO 1. Fatores que dificultam a adesão da mulher à realização do exame Papanicolau}

\begin{tabular}{|c|c|}
\hline AUTORES / ANO & ARTIGO \\
\hline $\begin{array}{l}\text { Alencar, Mendes, \& } \\
\text { Carvalho, } 2019\end{array}$ & $\begin{array}{l}\text { Dificuldades enfrentadas para realização do exame } \\
\text { ginecológico preventivo. }\end{array}$ \\
\hline Garcia et al., 2021 & $\begin{array}{l}\text { Identificação dos fatores que interferem na baixa } \\
\text { cobertura do rastreio do câncer de colo uterino através } \\
\text { das representações sociais de usuárias dos serviços } \\
\text { públicos. }\end{array}$ \\
\hline Marques, \& Paiva, 2019 & $\begin{array}{l}\text { Avaliação do perfil e da adesão ao colpocitológico de } \\
\text { mulheres em idade fértil. }\end{array}$ \\
\hline Melo et al., 2021 & $\begin{array}{l}\text { Percepção das mulheres acerca do exame de } \\
\text { Papanicolaou: revisão integrativa. }\end{array}$ \\
\hline Monteiro et al., 2019 & $\begin{array}{l}\text { A percepção de mulheres sobre o Papilomavírus } \\
\text { humano, o câncer do colo do útero e o exame de } \\
\text { Papanicolaou. }\end{array}$ \\
\hline Oliveira et al., 2020 & $\begin{array}{l}\text { A não realização do exame Papanicolaou e } \\
\text { comportamentos de risco em mulheres com vida sexual } \\
\text { ativa. }\end{array}$ \\
\hline Santos et al., 2020 & $\begin{array}{l}\text { Receptividade e Fatores Associados a Medidas } \\
\text { Preventivas ao Câncer de Colo do Útero em uma Capital } \\
\text { do Norte do Brasil. }\end{array}$ \\
\hline $\begin{array}{l}\text { Santos, Batista, } \\
\text { Oliveira, } 2020\end{array}$ & $\begin{array}{l}\text { Preditores da não adesão periódica ao exame } \\
\text { Papanicolau. }\end{array}$ \\
\hline Silva et al., 2020 & $\begin{array}{l}\text { Fatores relacionados a não adesão ao exame } \\
\text { citopatológico em mulheres na melhor idade: uma } \\
\text { revisão sistemática com Metassíntese. }\end{array}$ \\
\hline $\begin{array}{l}\text { Onofre, Vieir, \& Bueno, } \\
2019\end{array}$ & $\begin{array}{l}\text { Principais fatores que dificultam a adesão ao exame de } \\
\text { citologia oncótica: uma revisão de literatura. }\end{array}$ \\
\hline
\end{tabular}

Fonte: Dados da pesquisa, 2021.

prática sexual desprotegida (Oliveiraet al., 2020; Silvaet al., 2020). A prevenção primária consiste em fazer uso de preservativos e ser administrada a vacina contra HPV e, sua detecção precoce, depende da prevenção secundária que é a realização da coleta do exame Papanicolau onde será diagnosticado. A realização desse exame tem como público-alvo mulheres de 25 a 64 anos (Silvaet al., 2020). Esse exame á a principal ferramenta no rastreio do CCU.

Santos et al. (2020) informam que:

O exame PCCU é realizado em mulheres de 25 a 64 anos, sendo preconizada a repetição do exame a cada três anos após dois resultados normais consecutivos obtidos com um intervalo de um ano. Mesmo as mulheres vacinadas, quando alcançarem a idade preconizada, deverão realizar o PCCU, pois a vacina não protege contra todos os subtipos oncogênicos do HPV.

Diante disso, Onofre, Vieira e Bueno (2019) ressaltam que é muito importante que a vacinação e o exame preventivo caminhem juntos nas ações de prevenções, pois a vacina não protege contra todos os subtipos que causam o câncer. Para que haja um impacto epidemiológico na diminuição das taxas de incidência e mortalidade pelo CCU, estima-se que os programas de rastreamento devem cobrir $85 \%$ das mulheres (SANTOS et al., 2020).

Entretanto, Alencar, Mendes e Carvalho (2020), ressaltam que existem muitas barreiras enfrentadas pelas mulheres para realização do exame Papanicolau, o que torna de extrema importância o papel do profissional de saúde, frente a ações de prevenção da doença.

O Ministério da Saúde refere que a prevenção do câncer do colo uterino, na atenção integral à mulher, é uma prática do profissional enfermeiro, cabendo-lhe realizar: consulta de enfermagem, ações educativas, o exame preventivo e clínico das mamas, solicitação de exames complementares e prescrição de medicações, mediante protocolos ou normativas técnicas estabelecidas, observando disposições legais da profissão. E que

\section{RESULTADOS}

Medo e vergonha, desconforto e dor do procedimento. Difícil acesso à consulta, falta de vaga, local inadequado, inflexibilidade de horário para coleta, falta de privacidade e de humanização no acolhimento. Medo, vergonha e tabu, desinformação e desinteresse.

Raça, ocupação, renda familiar, escolaridade, situação conjugal, sexarca e histórico de abortamento associadas à adesão de prevenção de câncer de colo de útero.

Sentimento de vergonha, medo, o desconhecimento sobre importância da realização do exame.

Vergonha para realizar o exame, padrões culturais, desconhecimento, medo, negação de que pode vir a adoecer.

Medo, vergonha, insegurança, preconceito, falta de tempo.

Conhecimentos insuficientes sobre o HPV e sobre a vacina.

Vergonha, inflexibilidade de horários, dificuldade no agendamento, demora em receber o resultado, medo do resultado e da falta de ética profissional.

Difícil acesso, dificuldade para agendar o exame, demora no resultado e a falta de material, negligência e falta de tempo das participantes, o sentimento de insegurança, o receio de sentir dor e vergonha durante a realização do exame, problemas de comunicação entre as pacientes, profissionais e do serviço de saúde.

Medo, vergonha, cultura, baixa renda e baixa escolaridade, ausência de vínculo com o profissional, falta de preparo do profissional, desconhecimento sobre patologia e o exame.

posicioná-la de maneira confortável, compreendendo e informando cada etapa do procedimento submetida, visando não causar constrangimentos e diminuir ansiedade gerada pelo exame (Alencar et al., 2020, p.76).

De acordo com Soares, Pereira e Silva (2020), embora o exame de Papanicolau tenha sido introduzido no Brasil na década de 1940 e apesar das várias iniciativas governamentais, a cobertura de realização do exame ainda é baixa. Na opinião de Monteiro, Andrade e Vargas (2019) isso pode ser explicado por este assunto ainda ser tratado com um paradigma entre as mulheres, sendo este o principal motivo pelo qual elas tendem a não procurar assistência para sanar suas dúvidas sobre o assunto.

Nesse sentido, destaca-se o papel do profissional de saúde enquanto educador, podendo influenciar os comportamentos, atitudes e práticas da população, principalmente em relação ao exame de Papanicolau e outros que integram a saúde da mulher (Mesquitaet al., 2020). De acordo com Monteiro, Andrade e Vargas (2019) muitas pesquisas têm demonstrado a falta de conhecimento integral da população feminina sobre a importância e a finalidade do exame de Papanicolau, bem como, sobre os riscos que as infecções ocasionadas pelo vírus HPV podem trazer. Esses fatores diminuem a procura pelos serviços de saúde e refletem no aumento de casos de mulheres com diagnóstico tardio da doença. Nessa conjuntura, alcançar alta cobertura do exame de rastreamento da população-alvo é o componente mais importante para obter redução significativa da incidência e mortalidade por CCU, pois estima-se que de 12 a $20 \%$ das mulheres brasileiras de 25 a 64 anos de idade nunca tenham realizado o exame (Soares, Pereira \& Silva, 2020). Soares, Pereira e Silva (2020), apontam como estratégias de intervenção para aumentar a cobertura e acesso ao rastreamento do $\mathrm{CCU}$, a melhoria das informações fornecidas pelos profissionais de saúde às mulheres sobre a prevenção, tendo em vista que o maior conhecimento sobre o CCU e sua prevenção associa-se significativamente com a maior adesão à realização do exame de Papanicolau. Nesse ínterim, Onofre, Vieira e Bueno (2019) reforçam que é importante elaborar estratégias para lidar com o hábito das pacientes só buscarem o serviço quando estão doentes ou com alguma 
queixa. Para essas mesmas autoras, este tipo de comportamento está infundido na cultura brasileira e sua mudança depende de ações educativas que transformem os hábitos e valorizem a realidade dessas mulheres. Dessa forma, é de extrema importância o empenho das equipes de Saúde da Família para desenvolver atividades e ampliar os conhecimentos, respeitando a vida social, a história e a cultura das clientes, adotando um vocabulário inteligível para compartilhar saberes e condutas a serem tomadas para aquelas que não frequentam os serviços de saúde (Silvaet al., 2020).

\section{CONCLUSÃO}

Diante dos resultados apresentados foi possível identificar os principais fatores que dificultam a adesão da mulher à realização do exame preventivo ou Papanicolau, sendo eles: medo, vergonha, cultura, baixa renda, baixa escolaridade, falta de vínculo com o profissional, falta de preparo do profissional, desconhecimento sobre a patologia e o exame. O estudo evidenciou ainda que a falta de tempo, a demora na entrega dos resultados, a dificuldade de acesso, o preconceito, a superlotação de agendas, o sentimento de insegurança e a negação são também fatores que dificultam a adesão da mulher ao exame Papanicolau. A raça, ocupação, renda familiar, escolaridade, situação conjugal, sexarca e histórico de abortamento também foram associados à adesão de prevenção de câncer de colo de útero. Diante dos resultados apresentados, evidencia-se a importância da implementação de estratégiasna tentativa de minimizar tais fatores, como a capacitação do profissional de saúde, a realização de ações voltadas para a educação permanente em saúde e readequação do serviço de saúde de forma a melhorar o acesso. Nessa perspectiva, o profissional de saúde, em especial, o enfermeiro, deve conhecer a população feminina de forma a desenvolver a sensibilidade, a empatia e ter uma postura humanística perante essas pacientes. Deve-se ainda atentar para a realização de busca ativa das mulheres que necessitem fazer a consulta procurando desenvolver a formação de vínculo de confiança. Consequentemente a mulher se sentirá mais segura e orientada na hora de procurar uma forma de prevenção. Nesse sentido, considera-se de suma importância a implementação de ações de educação em saúde no âmbito da Estratégia de Saúde da Família (ESF) dispensando especial atenção ao público feminino, a fim de diminuir as lacunas existentes na sua procura pelo serviço e ainda, reduzir os fatores que influenciam na baixa adesão de mulheres ao exame preventivo ou Papanicolau.

\section{REFERÊNCIAS}

Alencar, M. L. S., Mendes, A. N., \& Carvalho, M. T. S. (2019). Dificuldades enfrentadas para realização do exame ginecológico preventivo. BrazilianJournalofSurgeryandClinicalResearch BJSCR, 26(1), 75-79. Disponível online em https://www. mastereditora.com.br/periodico/20190407_140613.pdf.

Garcia, M., Campos, G. K. P., Patrício, J. A. L., Rodrigues, A. L., \& Rodrigues, A. D. M.(2021). Identificação dos fatores que interferem na baixa cobertura do rastreio do câncer de colo uterino através das representações sociais de usuárias dos serviços públicos. BrazilianJournalof Health Review, 4(1), 1462-1477. Disponível online em https://www.brazilian journals.com/ index.php/BJHR/article/view/23271/18706.

Marconi, M. A. \&Lakatos, E. M. (2017). Fundamentos de metodologia científica. ( $8^{\mathrm{a}}$ ed). São Paulo: Atlas.
Marques, C. R., \& Paiva, A. C. (2019). Avaliação do perfil e da adesão ao colpocitológico de mulheres em idade fértil. BrazilianJournalofTechnology, Curitiba, 2(4), 984-997. Disponível online em: https://www.brazilianjournals.com/ index.php/BJT/article/view/6704/6361.

Melo, M. L. R. R., Silva, R. F. C., Oliveira, J. V. L., \& Lucena, M. A. (2021). Percepção das mulheres acerca do exame de Papanicolaou: revisão integrativa. Revista Eletrônica da Estácio, 6(2). Disponível online em https://reer.emnuvens.com.br/ reer/article/view/527.

Mesquita, A. D., Teles, K. K. N., Silva, S. C. B., Silva, F. R., Lima, L. K. C., Costa, R. S. L., \& Arruda, E. F. (2020).Conhecimentos, atitudes e práticas de mulheres frente ao exame preventivo do câncer do colo uterino. Journal Health NPEPS, 5(1), 261-275. Disponível online em https://periodicos.unemat.br/index.php/ jhnpeps/article/view/4184/3610.

Monteiro, C. M., Andrade, V. R. M., \& Vargas, F. A. (2019). A percepção de mulheres sobre o Papilomavírus humano, o câncer do colo do útero e o exame de Papanicolaou. Revista Interdisciplinar em Ciências da Saúde e Biológicas, 3(1), 23-31. Disponível online em https://core.ac.uk/download/pdf/ 322642414.pdf.

Oliveira, E. M. F., Oliveira, S. X., Caldas, M. L. L. S., Oliveira, M. B., Oliveira, M. X.,\& Lima, T. N. F. A. (2020)A não realização do exame Papanicolaou e comportamentos de risco em mulheres com vida sexual ativa. Revista Eletrônica Acervo Saúde, 12(12), e4221.Disponível online em: https://acervomais.com.br/ index.php/saude/article/view/4221.

Onofre, M. F., Vieira, R. D.,\&Bueno, G. H. (2019). Principais fatores que dificultam a adesão ao exame de citologia oncótica: uma revisão de literatura. Enfermagem Revista, 22(2), 231-242. Disponível online emhttp://periodicos.pucminas.br/index.php/ enfermagemrevista/article/view/21082.

Santos, A. P. A., Batista, A. A. V.,\&Oliveira, R. S.(2020). Preditores da não adesão periódica ao exame Papanicolau. Journalof Health Connections, 10(3),82-97. Disponível online em http://periodicos.estacio.br/index.php/journalhc/article/viewArti cle/6179.

Santos, N. L. F.; Valadares, L. R.; Melo, A. T.; Ferreira, J. C. G; Guimarães, M. S. A., Amaral, L. R. O. G., \&Mucari, T. B. (2020).Receptividade e fatores associados a medidas preventivas ao câncer de colo do útero em uma capital do Norte do Brasil. Mundo da Saúde, 1(44), 454-464. Disponível online em: $\quad$ https://revistamundodasaude.emnuvens.com.br/ mundodasaude/article/view/973/927.

Silva, M. D. T., Marques, R. B., \& Costa, L. O. Câncer de colo de útero: barreiras preventivas no século 21. (2021). BrazilianJournalof Health Review, 4(2), 7610-7626. Disponível em https://www.brazilianjournals.com/index.php/BJHR/ article/ view/27818/22017.

Silva, M. O., Souza, J. G., Martins, M. O., Ferraz, B. R., Barbosa, E. C.,\&Luz, D. C. R. P. (2020). Fatores relacionados a não adesão ao exame citopatológico em mulheres na melhor idade: uma revisão sistemática com metassíntese. Brazilian Journal of Development, 6(8), 60925-60934. Disponível online em: https://www.brazilianjournals.com/index.php/BRJD/article/view $/ 15442 / 12711$

Soares, M. B. O., Pereira, G. A., \& Silva, S. R. D. A. (2020). Fatores associados ao conhecimento sobre Papanicolaou. Ciência, Cuidado e Saúde, 19,e48557. Disponível online em https://periodicos.uem.br/ojs/index.php/CiencCuidSaude/article/ view/48557. 